\title{
Controle hormonal dos adenomas hipofisários pela cirurgia transesfenoidal: evolução dos resultados nos primeiros cinco anos de experiência
}

\author{
Hormonal control of pituitary adenomas by transsphenoidal \\ surgery: results of the first five years of experience
}

Erika Ribeiro Barbosa', Samuel Tau Zymberg'2, Rodrigo de Paula Santos ${ }^{3}$, Helio Rubens Machado ${ }^{4}$, Julio Abucham'

Disciplina de Endocrinologia, Escola Paulista de Medicina, Universidade Federal de São Paulo (Unifesp/EPM), São Paulo, SP, Brasil 2 Disciplina de Neurocirurgia, Unifesp/EPM, SP, Brasil

${ }^{3}$ Disciplina de Otorrinolaringologia, Unifesp/EPM, SP, Brasil

${ }_{4}^{4}$ Disciplina de Neurocirurgia, Faculdade de Medicina de Ribeirão Preto, Universidade de São Paulo (FMRP-USP). Ribeirão Preto, SP, Brasil
Correspondência para: Julio Abucham

Rua Pedro de Toledo, 910 04039-002 - São Paulo, SP, Brasil julioabucham@uol.com.br

Recebido em 10/08/2010 Aceito em 16/11/2010

\section{RESUMO}

Objetivo: Avaliar os resultados iniciais de uma equipe cirúrgica no controle hormonal dos adenomas hipofisários secretores. Materiais e métodos: Em cinco anos, foram operados 51 adenomas secretores (31 GH, $14 \mathrm{ACTH}, 5$ prolactina, $1 \mathrm{TSH}$ ). 0 controle hormonal foi $\mathrm{GH}$ basal $\leq 2,5 \mathrm{ng} / \mathrm{dL}$, cortisol livre urinário normal, redução dos níveis de prolactina, eT3 eT4 livre normais. Resultados: As taxas de controle foram $36 \%$ na acromegalia e $57 \%$ no Cushing. Dois prolactinomas (40\%) normalizaram a prolactina. Os hormônios tiroidianos normalizaram no adenoma secretor deTSH. $O$ controle do hipercortisolismo correlacionou-se com o tempo de experiência da equipe $(p=0,01)$. Conclusão: Nossos resultados, limitados aos primeiros anos de experiência cirúrgica, situam-se abaixo da variação reportada em grandes casuísticas com maior tempo de experiência. Ao longo do tempo, observou-se melhora progressiva nos níveis de cortisol urinário no pós-operatório inicial da doença de Cushing em função da experiência cirúrgica. Arq Bras Endocrinol Metab. 2011;55(1):16-28

\section{Descritores}

Cirurgia transesfenoidal; adenoma hipofisário; doença de Cushing; acromegalia; prolactinoma

\section{ABSTRACT}

Objective: To evaluate the initial results of a surgical team in the hormonal control of secreting pituitary adenomas. Materials and methods: In five years 51 functioning adenomas were operated (31 GH-secreting, $14 \mathrm{ACTH}$-secreting, $5 \mathrm{PRL}$-secreting and $1 \mathrm{TSH}$-secreting). Hormonal control was defined as $\mathrm{GH} \leq 2,5 \mathrm{ng} / \mathrm{mL}$, normal free-urinary cortisol, lower prolactin and normal T3 and FT4. Results: Control rates were $36 \%$ in acromegaly, and $57 \%$ in Cushing's disease. Two prolactinomas normalized prolactin levels. Thyroid hormone levels were normalized in theTSH-secreting adenoma. Control of hypercortisolism was positively correlated with years of experience $(p=0.01)$. Conclusion: Our results, although restricted to the beginning of our experience, lie below the reported range of other surgical series with much longer experience. During these years, there was a significant improvement in initial post surgery urinary cortisol levels in Cushing's disease as a function of surgical experience. Arq Bras Endocrinol Metab. 2011;55(1):16-28

Keywords

Transesphenoidal surgery; pituitary adenoma; Cushing's disease; acromegaly; prolactinoma

\section{INTRODUÇÃO}

A primeira cirurgia por via transesfenoidal bem-sucediAda foi realizada em 1907 por Herman Schloffer (1).
Entre 1910 e 1925, Harvey Cushing aprimorou a técnica e operou 231 pacientes usando incisão sublabial e acesso endonasal após retirada do septo (2). Durante 
as próximas décadas, essa técnica foi substituída pela craniotomia frontal, tendo em vista limitações como campo operatório estreito, iluminação deficiente e risco de infecção. A abordagem transesfenoidal para acesso à hipófise foi retomada na década de 1960, após a introdução do microscópio cirúrgico por Jules Hardy (3) e do intensificador de imagem, para confirmação da trajetória cirúrgica, por Gerard Guiot (4). Desde então, o acesso transeptal-transesfenoidal tem sido a abordagem de escolha nas cirurgias da região selar. Mais recentemente, avanços tecnológicos como microinstrumentos e óticas anguladas têm viabilizado a cirurgia endoscópica da região selar.

Além dos avanços tecnológicos, as características do tumor, como tamanho, consistência e invasividade, e a experiência do cirurgião são fatores determinantes no resultado dessa cirurgia. A partir de 2001, todas as cirurgias hipofisárias em pacientes da Unidade de Neuroendocrinologia da Disciplina de Endocrinologia da Escola Paulista de Medicina da Universidade Federal de São Paulo (EPM-Unifesp) foram executadas por uma equipe composta por um neurocirurgião e um otorrinolaringologista sob orientação inicial de um neurocirurgião experiente em cirurgia transeptal-transesfenoidal por via sublabial e auxílio de microscópio cirúrgico. A partir de 2003, essa via de acesso foi substituída pelo acesso transnasal endoscópico. O objetivo deste trabalho é avaliar a evolução dos resultados cirúrgicos dessa equipe no tratamento dos adenomas hipofisários secretores (GH, ACTH, prolactina e TSH) operados entre março de 2001 e março de 2006.

\section{PACIENTES E MÉTODOS}

\section{Pacientes}

Entre março de 2001 e março de 2006, o grupo de cirurgia hipofisária da EPM-Unifesp realizou 180 cirurgias em 156 pacientes com patologia da região selar (Tabela 1). Desses, foi selecionado para esse estudo um total de 51 pacientes portadores de adenomas secretores de GH $(\mathrm{n}=31)$, ACTH $(\mathrm{n}=14)$, prolactina $(\mathrm{n}=5)$ e TSH $(\mathrm{n}=1)$, submetidos a uma primeira intervenção cirúrgica pela equipe, com ou sem cirurgia prévia por outra equipe. Após aprovação do Comitê de Ética e Pesquisa da Universidade de São Paulo, todos os prontuários foram analisados retrospectivamente.
Tabela 1. Cirurgias hipofisárias realizadas entre março de 2001 e março de 2006

\begin{tabular}{lcc}
\hline Diagnóstico & Pacientes & Cirurgias \\
\hline Adenoma não secretor & $95(60,9 \%)$ & $113(63,3 \%)$ \\
Adenoma secretor de GH & $31(19,9 \%)$ & $33(18,2 \%)$ \\
Adenoma secretor de ACTH & $14(9 \%)$ & $17(9,4 \%)$ \\
Adenoma secretor de TSH & $1(0,6 \%)$ & $1(0,5 \%)$ \\
Adenoma secretor de prolactina & $5(3,2 \%)$ & $5(6,2 \%)$ \\
Craniofaringeoma & $4(2,6 \%)$ & $4(2,2 \%)$ \\
Apoplexia & $2(1,3 \%)$ & $2(1,0 \%)$ \\
Cisto de bolsa de Ratke & $2(1,3 \%)$ & $2(1,0 \%)$ \\
Metástase & $1(0,6 \%)$ & $2(1,0 \%)$ \\
Hipofisite linfocítica & $1(0,6 \%)$ & $1(0,5 \%)$ \\
Total & $156(100 \%)$ & $180(100 \%)$ \\
\hline
\end{tabular}

\section{Diagnóstico de acromegalia}

O diagnóstico de acromegalia foi baseado na suspeita clínica, confirmado pela presença de níveis elevados de IGF-I com níveis elevados de GH e/ou sem supressão após sobrecarga de glicose, na presença de lesão expansiva hipofisária na ressonância magnética. A campimetria visual foi realizada quando indicado. Os tumores eram classificados como microadenomas (tumor $\leq 10 \mathrm{~mm}$ ), macroadenomas circunscritos (tumor $>10 \mathrm{~mm}$, restrito à sela) ou macroadenomas invasivos (tumor $>10 \mathrm{~mm}$ com invasão de seio cavernoso e/ou esfenoidal).

\section{Diagnóstico de doença de Cushing}

O diagnóstico de doença de Cushing em um paciente com suspeita clínica de hipercortisolismo endógeno foi estabelecido pela presença de níveis elevados de cortisol livre em urina de 24 horas em pelo menos duas amostras, falta de supressão do cortisol sérico matinal (> 1,8 $\mathrm{ng} / \mathrm{dL}$ ) após $\mathrm{l} \mathrm{mg}$ de dexametasona na noite anterior e níveis normais ou elevados de ACTH pela manhã. Essa propedêutica foi geralmente complementada pelo teste do DDAVP para a presença de resposta do ACTH (> 35\%) e do cortisol (> 20\%) e pela ressonância magnética da região selar. Durante o período de cinco anos, o cortisol livre urinário foi dosado por RIA de dois kits diferentes, por isso os valores foram expressos como a razão entre a dosagem e o valor do limite superior da normalidade. Os tumores foram classificados como microadenomas (tumor $\leq 10 \mathrm{~mm}$ ) ou macroadenomas (tumor $>10 \mathrm{~mm}$ ). Somente um paciente não apresentou evidência de adenoma hipofisário na ressonância magnética e foi submetido ao cateterismo do seio petroso inferior para dosagem simultânea de ACTH central e periférico antes e após estímulo com DDAVP. 


\section{Diagnóstico de prolactinoma}

O diagnóstico de macroprolactinoma foi baseado na presença de níveis de prolactina $>200 \mathrm{ng} / \mathrm{ml} \mathrm{em} \mathrm{pa-}$ cientes com lesão expansiva hipofisária $>1,0 \mathrm{~cm}$ na ressonância magnética. Pacientes com macroadenomas hipofisários e níveis de PRL entre 100 e 200 ng/ml foram confirmados como prolactinomas apenas se a imuno-histoquímica fosse francamente positiva para PRL. Nenhum microprolactinoma foi operado.

\section{Diagnóstico de hipertiroidismo por adenoma secretor de TSH}

Um paciente com adenoma secretor de TSH apresentou quadro clínico compatível com tirotoxicose, com níveis séricos elevados de TSH, tiroxina livre, triiodotironina e subunidade $\alpha$ e um microadenoma hipofisário na ressonância magnética.

\section{Avaliação da função adeno-hipofisária}

A função adeno-hipofisária foi investigada em todos os pacientes por meio de dosagens hormonais séricas basais de hormônios periféricos e adeno-hipofisários relacionados aos outros eixos hormonais não primariamente envolvidos na hipersecreção tumoral $(\mathrm{GH}$, IGF-I, prolactina, TSH, T4 livre, cortisol, LH, FSH e testosterona total). Pacientes em amenorreia também foram consideradas hipogonádicas.

\section{Indicação cirúrgica}

Todos os pacientes tiveram a indicação cirúrgica definida em reunião multidisciplinar semanal da Unidade de Neuroendocrinologia da EPM, da qual participam endocrinologistas, radiologistas, neurocirurgiões e otorrinolaringologistas.

\section{Cirurgia}

Todas as cirurgias foram realizadas no Hospital São Paulo. Inicialmente, contou-se com a orientação do Dr. Helio Rubens Machado, professor do Departamento de Neurocirurgia da Faculdade de Medicina de Ribeirão Preto da Universidade de São Paulo e professor visitante de Neurocirurgia na Escola Paulista de Medicina da Universidade Federal de São Paulo entre os anos 2001 e 2003. Até o início de 2002, todas as cirurgias ( $\mathrm{n}=14$ ) foram realizadas com auxílio de microscópio cirúrgico e a via de acesso foi sublabial. Depois, nas 37 cirurgias restantes, a via passou a ser transnasal, com auxílio de endoscópio. No pós-operatório imediato, todos os pacientes foram encaminhados à enfermaria de Endocrinologia, sem necessidade de internação em Unidade de Terapia Intensiva.

\section{Avaliação pós-operatória e critérios de controle hormonal}

Todos os pacientes foram reavaliados no pós-operatório por meio de dosagens hormonais e ressonância magnética. As dosagens hormonais eram, em média, realizadas 2,4 $\pm 1,8$ meses após a cirurgia (mediana: 2 meses; intervalo: 1-8 meses). Os níveis hormonais considerados para se definir o controle hormonal pela cirurgia foram: acromegalia: $\mathrm{GH} \leq 2,5 \mathrm{ng} / \mathrm{mL}$ (média de 2 dosagens em dias diferentes) e/ou IGF-I expressa como a razão entre a dosagem e o valor do limite superior da normalidade para o sexo e idade (normal: $\leq 1$; média de 2 dosagens em dias diferentes). Doença de Cushing: cortisol livre urinário de 24 horas normal (média de 2 dosagens em dias diferentes), expresso como a razão entre a dosagem e o valor do limite superior da normalidade (normal: $\leq 1$ ). Prolactinoma: normalização dos níveis de prolactina com doses iguais ou menores de agonistas dopaminérgicos que as usadas no pré-operatório. Adenoma secretor de TSH: T3 total e T4 livre normais.

\section{Dosagens hormonais}

Todas as dosagens hormonais foram realizadas em $\mathrm{du}^{-}$ plicata. GH: ensaio por quimioluminescência (GH Immulite 2000). Sensibilidade: $0,01 \mathrm{ng} / \mathrm{mL}$. Coeficientes de variação intraensaio e interensaio: $2,9-4,6 \%$ e 4,2$6,6 \%$, respectivamente. VN: 0-2,5 ng/mL. IGF-I: ensaio imunorradiométrico (DSL, Texas, USA). Sensibilidade: $0,8 \mathrm{ng} / \mathrm{mL}$. Coeficientes de variação intraensaio e interensaio: $1,5-3,4 \%$ e $1,5-8,2 \%$, respectivamente. TSH: ensaio por quimioluminescência (Advia-Centaur). Sensibilidade: $0,01 \mathrm{ng} / \mathrm{mL}$. Coeficientes de variação intraensaio e interensaio: 2,0-5,3\% e 2,1-2,5\%, respectivamente. VN: $0,35-5,5 \mathrm{U} / \mathrm{dL}$. T4 livre: ensaio imunofluorimétrico (Delfia Wallac Oy, Turku, Finland). Sensibilidade: $0,16 \mathrm{ng} / \mathrm{dL}$. Coeficientes de variação intraensaio e interensaio: $4,4 \%$ e $6,1 \%$, respectivamente. VN: $0,7-1,54 \mathrm{ng} / \mathrm{dL}$. Cortisol sérico: ensaio por quimioluminescência (Advia-Centaur). Sensibilidade: 0,2 $\mathrm{mcg} / \mathrm{dL}$. Coeficientes de variação intraensaio e interensaio: $2,9-3,8 \%$ e $1,9-5,4 \%$, respectivamente. $\mathrm{VN}$ : 4,3- 
$22,4 \mathrm{mcg} / \mathrm{dL}$. Cortisol livre urinário: radioimunoensaio (DSL 2100 Active Genese, Texas, USA). Sensibilidade: $0,3 \mathrm{mcg} / \mathrm{dL}$. Coeficientes de variação intraensaio e interensaio: $9,5 \%$ e $8,4 \%$, respectivamente. $\mathrm{VN}$ : $34-122$ $\mathrm{mcg} / 24 \mathrm{~h}$ e ensaio quimioluminescência (Advia-Centaur). Sensibilidade: $0,2-75 \mathrm{mcg} / \mathrm{dL}$. Coeficientes de variação intraensaio e interensaio: 2,9-3,8\% e 1,9-5,4\%, respectivamente. VN: $28,5-213,7 \mathrm{mcg} / 24 \mathrm{~h}$. ACTH: ensaio imuno-quimioluminométrico (Diagnostic Prod. Corporation, Los Angeles, USA). Sensibilidade: $5 \mathrm{pg} / \mathrm{mL}$. Coeficientes de variação intraensaio e interensaio: $3,6 \%$ e $2,8 \%$, respectivamente. VN: $20-70 \mathrm{pg} / \mathrm{mL}$. LH: ensaio quimioluminescência (Advia-Centaur). Sensibilidade: $0,07 \mathrm{UI} / \mathrm{L}$. Coeficientes de variação intraensaio e interensaio: $2,3-3,0 \%$ e 1,5-2,9\%, respectivamente. $\mathrm{VN}$ : 1,5-9,3 UI/L (homens); 1,9-12,5 UI/L (fase folicular); 8,7-76,3 UI/L (pico ovulatório); 0,5-16,9 UI/L (fase lútea). FSH: ensaio quimioluminescência (AdviaCentaur). Sensibilidade: 0,3-200 UI/L. Coeficientes de variação intraensaio e interensaio: $2,0-2,9 \%$ e 0,3 $2,7 \%$, respectivamente. VN: 1,4-18,1 UI/L (homens); 2,5-10,2 UI/L (fase folicular); 3,2-33,4 UI/L (pico ovulatório); 1,5-9,1 UI/L (fase lútea). Testosterona: ensaio imuno-quimioluminométrico (Immulite 2000). Sensibilidade: $20 \mathrm{ng} / \mathrm{dL}$. Coeficientes de variação intraensaio e interensaio: 2,3-3,0\% e 1,5-2,9\%, respectivamente. VN: 262-1593 ng/dL. Prolactina: ensaio por quimioluminescência (Advia-Centaur). Sensibilidade: $0,3 \mathrm{ng} / \mathrm{mL}$. Coeficientes de variação intraensaio e interensaio: $1,9-4,4 \%$ e $2,0-4,9 \%$, respectivamente. $\mathrm{VN}$ : 2-30 ng/L.

\section{Análise estatística}

Os resultados foram expressos em média \pm DP ou mediana \pm DP e percentuais. A análise estatística foi realizada com testes paramétricos ou não paramétricos, de acordo com resultados preliminares dos testes de normalidade. Para variáveis categóricas, foi usado o teste exato de Fisher ou o teste do qui-quadrado, conforme indicado. Dados contínuos com distribuição normal foram analisados por teste $t$ e correlação de Pearson. Dados contínuos com distribuição não normal foram analisados por Wilcoxon, Mann-Whitney e correlação de Spearman. Valores de $\mathrm{p}<0,05$ foram considerados significantes. A análise estatística foi realizada usando o programa GraphPad Prism versão 4.03 para Windows (GraphPad Software, San Diego California USA, www. graphpad.com.)

\section{RESULTADOS}

\section{Acromegalia}

Casuística: foram avaliados 31 pacientes com acromegalia, antes da primeira intervenção cirúrgica no serviço, com média de idade de 46,4 $\pm 10,5$ anos (mediana: 47,5 anos; intervalo: 20-65 anos), 23 do sexo feminino e 8 do sexo masculino. $\mathrm{Na} \mathrm{RM}$, cinco apresentaram microadenomas (16\%) e 26 apresentaram macroadenomas (84\%), sendo 11 macroadenomas circunscritos (36\%) e 15 invasivos (48\%). Quatro pacientes já haviam sido submetidos a uma cirurgia transesfenoidal em outros serviços. Nenhum paciente havia sido submetido à radioterapia. Após avaliação hormonal e radiológica, dez pacientes $(32 \%)$ foram tratados com análogos da somatostatina no pré-operatório: cinco com octreotida subcutânea e cinco com octreotida de depósito (Sandostatin-LAR ${ }^{\circledR}$ ). Nestes últimos, a reavaliação hormonal foi feita após dois meses ou mais da cirurgia. Todos os 31 pacientes foram reavaliados após uma primeira intervenção cirúrgica pela equipe de cirurgia hipofisária da Unifesp. O exame anatomopatológico confirmou adenoma com imuno-histoquímica positiva para $\mathrm{GH}$ em todos os pacientes.

GH: o nível médio de GH basal (Figura 1) caiu significativamente $(\mathrm{p}<0,04)$ após a cirurgia, de $32,3 \pm$ $42,8 \mathrm{ng} / \mathrm{mL}$ (mediana: $8,5 \mathrm{ng} / \mathrm{mL}$; intervalo: 1,6-223 $\mathrm{ng} / \mathrm{mL}$ ) para $7,8 \pm 10,4 \mathrm{ng} / \mathrm{mL}$ (mediana: $3,0 \mathrm{ng} /$ $\mathrm{mL}$; intervalo: $0,1-40 \mathrm{ng} / \mathrm{mL}$ ).

IGF-I: o nível de IGF-I (Figura 2), calculado em relação ao limite superior da normalidade (LSN) para sexo e idade (VN: $\leq 1,0)$, caiu significativamente $(\mathrm{p}<$ 0,04 ) após a cirurgia, de 4,0 $\pm 5,0$ (mediana: 2,3 ; intervalo: $1,2-24,7$ ) para $2,1 \pm 1,3$ (mediana: 2,0 ; intervalo: 0,6-5,8).

Função adeno-hipofisária: antes da cirurgia, um paciente apresentava pan-hipopituitarismo (3\%), uma apenas hipotiroidismo central $(3 \%)$ e nove apresentavam hipogonadismo hipogonadotrófico (29\%). Após a cirurgia, o único paciente com pan-hipopituitarismo no pré-operatório recuperou totalmente a função adeno-hipofisária. O único paciente com hipotiroidismo central isolado também recuperou a função tiroidiana. Dos nove pacientes com deficiência gonadotrófica, três recuperaram $(33 \%)$, cinco mantiveram a deficiência $(55 \%)$ e um adquiriu outras deficiências hormonais (11\%). Um paciente com função adeno-hipofisária normal no pré-operatório evoluiu com hipotiroidismo e hipogonadismo após a cirurgia (11\%). 


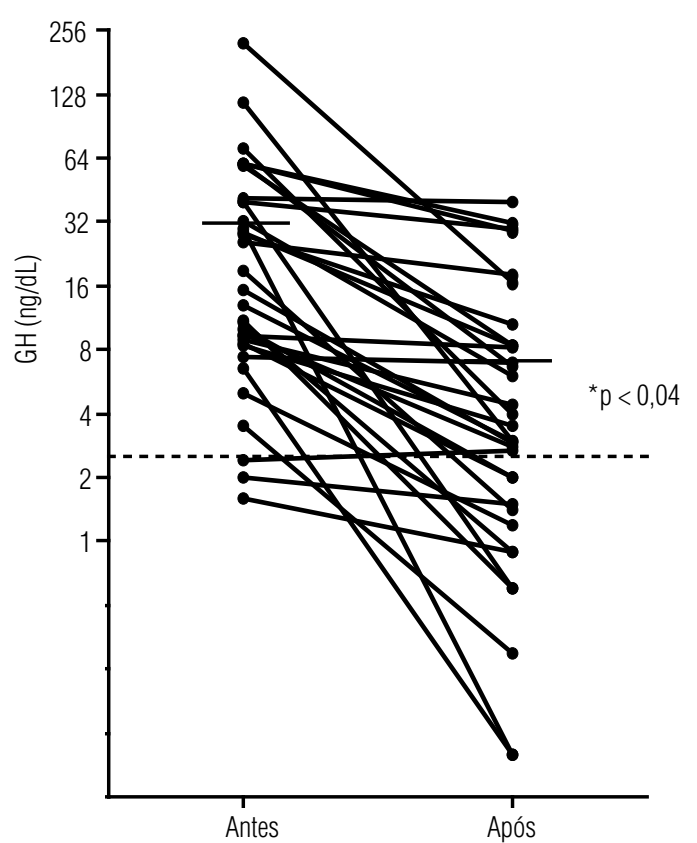

Figura 1. Níveis de GH (ng/dL) em pacientes acromegálicos antes e após cirurgia transesfenoidal.

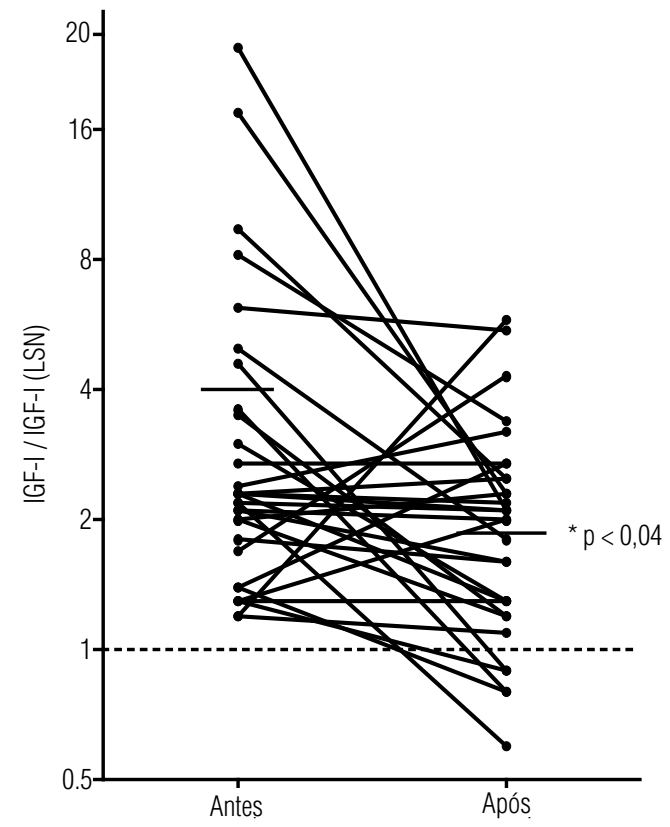

Figura 2. Níveis de IGF-I/IGF-I (LSN) em pacientes acromegálicos antes e após cirurgia transesfenoidal.

Visão: um paciente apresentava amaurose bilateral $(3 \%)$, três apresentavam hemianopsia bilateral $(10 \%)$ e um, hemianopsia à direita (3\%). Desses 5 pacientes, dois obtiveram recuperação parcial $(40 \%)$ e três obtiveram recuperação total da visão $(60 \%)$. Um paciente portador de macroadenoma invasivo apresentou piora do campo visual no pós-operatório, com quadrantoanopsia superior bilateral.
Outras complicações: não houve nenhum caso de meningite ou óbito perioperatório. Quatro pacientes apresentaram fístula liquórica (13\%), mas somente um necessitou drenagem lombar, não sendo necessária a reintervenção cirúrgica. Outros três pacientes apresentaram sangramento nasal (10\%), resolvido apenas com tamponamento nasal anterior. Diabetes insípido transitório ocorreu em quatro pacientes (13\%), todos com resolução do quadro em até 48 horas. Nenhum paciente apresentou diabetes insípido permanente.

\section{Classificação do controle hormonal após a cirurgia conforme GH e/ou IGF-I (Tabela 2)}

Grupo I: $\mathbf{G H} \leq \mathbf{2 , 5} \mathbf{n g} / \mathrm{mL}$ e IGF-I normal ( $\mathrm{n}=\mathbf{5}$ )

Cinco dos 31 pacientes operados (16\%) atingiram esse critério: 1 de 5 com microadenomas (20\%) e 4 de 26 com macroadenomas (15\%), sendo 3 de 11 com macroadenomas circunscritos (27\%) e 1 de 15 com macroadenomas invasivos (7\%). Nesse grupo, o GH médio pré-operatório foi $12,7 \pm 10,0 \mathrm{ng} / \mathrm{mL}$ (mediana: 10,8 $\mathrm{ng} / \mathrm{mL}$; intervalo: $5,0-30 \mathrm{ng} / \mathrm{mL}$ ) e a IGF-I foi $2,6 \pm$ 1,4 (mediana: 2,2 ; intervalo: $1,3-4,6$ ), caindo para 0,6 $\pm 0,5 \mathrm{ng} / \mathrm{mL}$ (mediana: $0,6 \mathrm{ng} / \mathrm{mL}$; intervalo: $0,1-1,2$ $\mathrm{ng} / \mathrm{mL} ; \mathrm{p}=0,03$ ) e $0,8 \pm 0,1$ (mediana: 0,8 ; intervalo: $0,6-0,9 ; \mathrm{p}=0,008)$, respectivamente, após a cirurgia.

Grupo II: GH $\leq 2,5 \mathrm{ng} / \mathrm{mL}$ ou IGF-I normal $(\mathrm{n}=6)$ Seis dos 31 pacientes operados (19\%) atingiram esse critério, todos com GH $\leq 2,5 \mathrm{ng} / \mathrm{mL}$ e IGF-I ainda elevada: 1 de 5 com microadenomas $(20 \%)$ e 5 de 26 com macroadenomas (19\%), sendo 3 de 11 com macroadenomas circunscritos (27\%) e 2 de 15 com macroadenomas invasivos (13\%). Nenhum paciente apresentou IGF-I normal e GH $>2,5 \mathrm{ng} / \mathrm{mL}$. Nesse grupo, o GH médio pré-operatório foi $13,5 \pm 14,4 \mathrm{ng} / \mathrm{mL}$ (mediana: $9,3 \mathrm{ng} / \mathrm{mL}$; intervalo: $1,6-40 \mathrm{ng} / \mathrm{mL}$ ) e a IGF-I foi 3,6 $\pm 2,5$ (mediana: 2,3 ; intervalo: $1,8-8,2$ ), caindo para $1,4 \pm 0,6 \mathrm{ng} / \mathrm{mL}$ (mediana: $1,5 \mathrm{ng} / \mathrm{mL}$; intervalo: $0,6-2,0 \mathrm{ng} / \mathrm{mL} ; \mathrm{p}=0,01$ ) e $2,1 \pm 0,8$ (mediana: 2,0 ; intervalo: $1,3-3,4 ; \mathrm{p}=0,18)$, respectivamente, após a cirurgia.

Grupo III: redução de GH $\geq \mathbf{5 0} \%$ e IGF-I elevada ( $n=13$ ) Treze dos 31 pacientes ( $42 \%$ ) atingiram esse critério: 1 de 5 com microadenomas (20\%) e 12 de 26 com macroadenomas (46\%), sendo 5 de 11 macroadenomas circunscritos (45\%) e 7 de 15 macroadenomas invasivos (47\%). Nesse grupo, o GH médio pré-operatório foi 
Tabela 2. Níveis de GH e IGF-1 antes e após cirurgia transesfenoidal em pacientes acromegálicos agrupados conforme controle hormonal obtido

\begin{tabular}{|c|c|c|c|c|c|c|c|}
\hline \multirow{2}{*}{ Grupo* } & \multirow{2}{*}{$\mathbf{n}$} & \multicolumn{3}{|c|}{$\mathrm{GH}(\mathrm{ng} / \mathrm{mL})$} & \multicolumn{3}{|c|}{ IGF-I / IGF-I (LSN**) } \\
\hline & & Antes & Após & $\mathbf{p}$ & Antes & Após & $\mathbf{p}$ \\
\hline I & 5 & $12,7 \pm 10,0$ & $0,6 \pm 0,5$ & 0,03 & $2,6 \pm 1,4$ & $0,8 \pm 0,1$ & 0,008 \\
\hline$\|$ & 6 & $13,5 \pm 14,4$ & $1,4 \pm 0,6$ & 0,01 & $3,6 \pm 2,5$ & $2,1 \pm 0,8$ & 0,18 \\
\hline III & 13 & $54,2 \pm 60,1$ & $8,1 \pm 7,3$ & $<0,001$ & $3,4 \pm 4,8$ & $2,1 \pm 1,0$ & 0,74 \\
\hline IV & 7 & $26,5 \pm 21,4$ & $19,3 \pm 14,6$ & 0,53 & $5,7 \pm 8,5$ & $3,2 \pm 1,8$ & 0,9 \\
\hline
\end{tabular}

* Grupo I: GH $\leq 2,5 \mathrm{ng} / \mathrm{mL}$ e IGF-I normal; Grupo II: GH $\leq 2,5 \mathrm{ng} / \mathrm{mL}$ ou IGF-I normal; Grupo III: GH > 2,5 ng/mL e redução de GH $\geq 50 \%$ e IGF-I elevada; Grupo IV: Nenhum dos critérios acima.

** LSN: IGF-I calculado em relação ao limite superior da normalidade para sexo e idade (VN: $\leq 1,0)$.

$54,2 \pm 60,1 \mathrm{ng} / \mathrm{mL}$ (mediana: $32,4 \mathrm{ng} / \mathrm{mL}$; intervalo: $7,4-223 \mathrm{ng} / \mathrm{mL}$ ) e a IGF-I foi $3,4 \pm 4,8$ (mediana: $2,1$; intervalo: $1,2-18,5)$, caindo para $8,1 \pm 7,3 \mathrm{ng} /$ $\mathrm{mL}$ (mediana: $6,0 \mathrm{ng} / \mathrm{mL}$; intervalo: $2,8-28,8 \mathrm{ng} / \mathrm{mL}$; $\mathrm{p}=0<0,001$ ) e $2,1 \pm 1,0$ (mediana: 2,0 ; intervalo: $1,1-4,3 ; \mathrm{p}=0,74)$, respectivamente, após a cirurgia.

\section{Grupo IV: GH e IGF-I não atingem os critérios} anteriores $(\mathbf{n}=7)$

Sete dos 31 pacientes $(22 \%)$ apresentaram GH $>2,5$ ng/dL sem redução de $\mathrm{GH} \geq 50 \%$ após a cirurgia: 2 entre os 5 pacientes com microadenomas $(40 \%)$ e 5 entre os 26 com macroadenomas (19\%), sendo todos macroadenomas invasivos. Nenhum desses pacientes apresentou IGF-I normal. Neste grupo, o GH médio pré-operatório foi $26,5 \pm 21,4 \mathrm{ng} / \mathrm{mL}$ (mediana: 25,7 $\mathrm{ng} / \mathrm{mL}$; intervalo: $2,4-60 \mathrm{ng} / \mathrm{mL}$ ) e a IGF-I foi $5,7 \pm$ 8,5 (mediana: 2,3; intervalo: 1,2-24,7) caindo para 19,3 $\pm 14,6 \mathrm{ng} / \mathrm{mL}$ (mediana: $17,9 \mathrm{ng} / \mathrm{mL}$; intervalo: 2,7 $40 \mathrm{ng} / \mathrm{mL} ; \mathrm{p}=0,53$ ) e $3,2 \pm 1,8$ (mediana: 2,7 ; intervalo: $1,2-5,8 ; \mathrm{p}=0,9)$, respectivamente, após a cirurgia.

Fatores preditivos da resposta cirúrgica $(\mathrm{GH})$ : conforme apresentado na tabela 3 , comparando-se os 11 pacientes que atingiram controle hormonal após a cirurgia (Grupos I e II) com os 20 que não atingiram (Grupos III e IV), observou-se que o nível de GH no diagnóstico foi significativamente menor nos pacientes controlados $(12,2 \pm 11,9 \mathrm{ng} / \mathrm{dL}$, intervalo: $0,3-40,0$ $\mathrm{ng} / \mathrm{dL}$ vs. $43,7 \pm 49,9 \mathrm{ng} / \mathrm{dL}$, intervalo: $2,4-223 \mathrm{ng} /$ $\mathrm{dL} ; \mathrm{p}=0,008$; Mann-Whitney).

Além disso, o controle hormonal após a cirurgia tendeu a ocorrer com maior frequência nos pacientes com tumores circunscritos $(8 / 16$ vs. $3 / 15, \mathrm{p}=0,13)$. Não houve diferença significativa em relação aos outros fatores analisados: idade $(\mathrm{p}=0,24)$, tratamento cirúrgico ( $\mathrm{p}=0,66)$ ou farmacológico prévio $(\mathrm{p}=0,60)$, proporção de micro/macroadenomas $(\mathrm{p}=1,0)$, técnica cirúrgica empregada (microscopia vs. endoscopia, $\mathrm{p}=0,66$ )
Tabela 3. Comparação entre nível pré-operatório de GH e IGF-I, idade, tratamentos prévios, tamanho tumoral, uso de microscopia ou endoscopia na cirurgia em acromegálicos controlados (grupos I e II) e não controlados (grupos III e IV)

\begin{tabular}{lccc}
\hline Caracteristica & $\begin{array}{c}\text { Controlados } \\
\mathbf{( 1 1 )}\end{array}$ & $\begin{array}{c}\text { Não } \\
\text { controlados } \\
\mathbf{( 2 0 )}\end{array}$ & p \\
\hline GH pré-operatório $(\mathrm{ng} / \mathrm{dL})$ & $12,2 \pm 11,9$ & $43,7 \pm 49,9$ & 0,008 \\
IGF-I pré-operatório $\left(\mathrm{LSN}^{\star}\right)$ & $3,6 \pm 2,3$ & $5,1 \pm 6,6$ & 0,92 \\
Idade & $50,7 \pm 10,3$ & $43,4 \pm 9,7$ & 0,24 \\
Cirurgia prévia & 2 & 2 & 0,66 \\
Sandostatin prévio & 1 & 9 & 0,6 \\
Micro: macroadenomas & $2: 9$ & $3: 17$ & 1,0 \\
Microscopia: endoscopia & $2: 9$ & $2: 18$ & 0,66 \\
\hline
\end{tabular}

* LSN: IGF-I calculado em relação ao limite superior da normalidade para sexo e idade (VN: $\leq 1,0)$.

ou ano em que foi realizada a cirurgia $(\mathrm{p}=0,56)$ entre pacientes controlados e não controlados pela cirurgia.

Evolução dos resultados cirúrgicos na acromegalia: nessa análise, os pacientes foram divididos em relação à época em que foram operados: 17 entre março de 2001 e setembro de 2003 e 14 entre outubro de 2003 e março de 2006. Foram considerados controlados $(\mathrm{GH} \leq 2,5 \mathrm{ng} / \mathrm{mL}) 7$ dos 17 pacientes (41\%) operados na primeira metade desses 5 anos e 4 dos 14 pacientes (29\%) operados na segunda metade desse período $(\mathrm{p}=$ $0,7)$. Analisando-se os resultados conforme o tamanho e a circunscrição dos adenomas, foram controlados 2 de 4 microadenomas ( $50 \%$ ) operados na primeira metade vs. 0 de $1(0 \%)$ na segunda metade do período $(\mathrm{p}=1)$, e 5 de 13 macroadenomas $(39 \%)$ na primeira metade vs. 4 de $13(31 \%)$ na segunda $(\mathrm{p}=1)$, sendo 3 de 5 macroadenomas circunscritos $(60 \%)$ na primeira metade vs. 3 de $6(50 \%)$ na segunda $(\mathrm{p}=1)$, e 2 de 8 macroadenomas invasivos $(25 \%)$ na primeira metade $v s$. 1 de $7(14 \%)$ na segunda $(\mathrm{p}=1)$. Nenhuma dessas análises comparativas mostrou diferença significativa entre os resultados obtidos nos dois períodos. 


\section{Doença de Cushing}

Casuística: foram avaliados 14 pacientes com doença de Cushing, antes da primeira intervenção por essa equipe cirúrgica, com idade média de 30,2 \pm 9,3 anos (mediana: 31,5 anos; intervalo: 13-40 anos), 12 do sexo feminino e 2 do sexo masculino. Na RM, 8 pacientes apresentavam microadenomas $(57 \%)$ e 5 macroadenomas $(36 \%)$. Um único paciente $(7 \%)$, sem evidência de lesão hipofisária, foi submetido a cateterismo bilateral do seio petroso inferior para dosagem de ACTH basal e após estímulo com desmopressina. O gradiente central/periferia foi 1,8 (basal) e 40 (após desmopressina), com lateralização à direita. Um paciente $(7 \%)$ já havia sido submetido a uma cirurgia transesfenoidal, por outro cirurgião, em outro serviço. Nenhum paciente havia sido submetido à radioterapia. Nove pacientes $(64 \%)$ receberam cetoconazol antes da cirurgia. O estudo anatomopatológico confirmou adenoma com imuno-histoquímica positiva para ACTH em todos os pacientes.

Cortisol livre urinário: no pré-operatório, o cortisol livre urinário foi $4,1 \pm 3,3$ vezes acima do limite superior da normalidade (mediana: 3,1 ; intervalo: 1,1 $13,1)$. Após a cirurgia, o cortisol livre urinário caiu significativamente $(\mathrm{p}=0,003)$ para $1,7 \pm 2,3$ vezes acima do limite superior da normalidade (mediana: 0,75 ; intervalo: 0-7,4) (Figura 3).

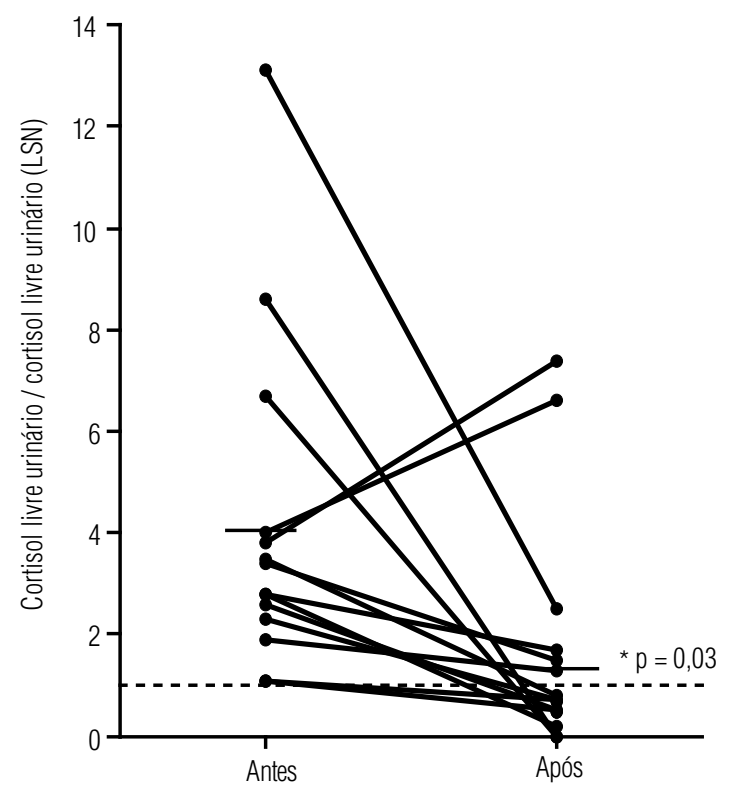

LSN: cortisol livre urinário calculado em relação ao limite superior da normalidade (VN: $\leq 1,0)$.

Figura 3. Níveis de cortisol livre urinário em pacientes com doença de Cushing antes e após cirurgia transesfenoidal.
ACTH: no pré-operatório, o ACTH foi 91,2 \pm 85,2 (mediana: 63, $1 \mathrm{pg} / \mathrm{dL}$; intervalo: 32,6-370 pg/dL). Após a cirurgia, o ACTH reduziu significativamente ( $\mathrm{p}$ $=0,01$ ) para 43,2 $\pm 34,1$ (mediana: $32,7 \mathrm{pg} / \mathrm{dL}$; intervalo: $11-121 \mathrm{pg} / \mathrm{dL}$ ).

Função adeno-hipofisária: seis pacientes do sexo feminino apresentaram amenorreia. Entre os dois pacientes do sexo masculino, um apresentou atraso no desenvolvimento puberal aos 15 anos. Nenhum paciente apresentou hipotiroidismo. Após a cirurgia, 7 pacientes (100\%) recuperaram a função gonadotrófica e 4 pacientes $(29 \%)$ desenvolveram hipotiroidismo.

Visão: nenhum paciente apresentou deficiência visual antes ou após a cirurgia.

Complicações: nenhum paciente apresentou meningite, sangramento ou fístula liquórica. Quatro pacientes apresentaram diabetes insípido transitório (29\%), todos com resolução em até 48 horas. Nenhum paciente evoluiu com diabetes insípido permanente. Nenhum óbito ocorreu.

Controle hormonal: o hipercortisolismo foi controlado pela cirurgia em oito pacientes $(57 \%)$, conforme avaliado pelo cortisol livre urinário. Quando consideradas as características radiológicas do tumor, 5 dos 8 microadenomas (55\%) e 3 dos 5 macroadenomas (60\%) foram controlados pela cirurgia. O paciente sem evidência radiológica de tumor hipofisário não foi controlado pela cirurgia, apesar de um microadenoma ter sido visualizado no procedimento. Entre os 9 pacientes que usaram cetoconazol no pré-operatório, 7 foram controlados pela cirurgia $(77 \%)$, dos quais 2 apresentaram insuficiência adrenal aguda no pós-operatório.

No grupo considerado controlado pela cirurgia $(\mathrm{n}=$ 8 ), o cortisol urinário caiu de $3,6 \pm 2,7$ vezes o limite superior da normalidade (mediana: 2,7 ; intervalo: $1,1-8,6$ ) no pré-operatório para $0,4 \pm 0,3 \mathrm{vez}$ o limite superior da normalidade (mediana: 0,5; intervalo: $0-0,8$ ) após a cirurgia $(\mathrm{p}<0,001)$, enquanto o ACTH caiu de $73 \pm$ 29,6 pg/dL (mediana: $64 \mathrm{pg} / \mathrm{dL}$; intervalo: 32,6-130 $\mathrm{pg} / \mathrm{dL}$ ) no pré-operatório para $28,9 \pm 22,5$ (mediana: 23,9; intervalo: $11,2-80)$ após a cirurgia $(\mathrm{p}=0,004)$.

No grupo não controlado pela cirurgia $(n=6)$, o cortisol urinário caiu de 4,8 \pm 4 , l vezes o limite superior da normalidade (mediana: 3,6; intervalo: 1,9-13,1) para 3,5 $\pm 2,7$ vezes o limite superior da normalidade (mediana: 2,1 ; intervalo: $1,3-7,4)$ após a cirurgia $(\mathrm{p}=$ $0,3)$, enquanto o ACTH caiu de $115,5 \pm 128,1 \mathrm{pg} / \mathrm{dL}$ (mediana: $61 \mathrm{pg} / \mathrm{dL}$; intervalo: $34,7-370 \mathrm{pg} / \mathrm{dL}$ ) para $62,3 \pm 39,4$ (mediana: 51 ; intervalo: $12-121$ ) após a cirurgia $(\mathrm{p}=0,58)($ Tabela 4$)$. 
Tabela 4. Níveis de cortisol livre urinário e ACTH em pacientes com doença de Cushing antes e após cirurgia transesfenoidal conforme controle hormonal obtido

\begin{tabular}{|c|c|c|c|c|c|c|}
\hline \multirow[b]{2}{*}{ Controle hormonal } & \multicolumn{3}{|c|}{ Cortisol urinário (LSN*) } & \multicolumn{3}{|c|}{ ACTH (pg/dL) } \\
\hline & Antes & Após & $\mathbf{P}$ & Antes & Após & $\mathbf{p}$ \\
\hline Controlados (8) & $3,6 \pm 2,7$ & $0,4 \pm 0,3$ & $<0,001$ & $73 \pm 29,6$ & $28,9 \pm 22,5$ & 0,3 \\
\hline Não controlados (6) & $4,8 \pm 4,1$ & $3,5 \pm 2,7$ & 0,3 & $115,5 \pm 128,1$ & $62,3 \pm 39,4$ & 0,58 \\
\hline
\end{tabular}

* LSN: cortisol livre urinário calculado em relação ao limite superior da normalidade (VN: $\leq 1,0)$.

Fatores preditivos da resposta cirúrgica: o grupo de pacientes controlados após a cirurgia foi comparado ao grupo não controlado em relação a características clínicas e laboratoriais. Não houve diferença entre os grupos de pacientes controlados e não controlados em relação ao cortisol urinário pré-operatório $(3,6 \pm 2,7$ vs. $4,8 \pm 4,1 ; \mathrm{p}=0,34$; Mann-Withney), ACTH pré-operatório $(73,0 \pm 29,6 v s .115,5 \pm 128,1 ; \mathrm{p}=0,75$; Mann-Withney), a proporção de micro/macroadenomas $(\mathrm{p}=1,0)$, técnica cirúrgica empregada (microscopia $v s$. endoscopia, $\mathrm{p}=0,66)$ ou idade $(34,8 \pm 6,4$ anos vs. $24,0 \pm 9,4 \mathrm{p}=0,07)$ (Tabela 5).

Tabela 5. Comparação entre nível pré-operatório de cortisol livre urinário e ACTH, idade, tamanho tumoral, uso de microscopia ou endoscopia na cirurgia em pacientes com doença de Cushing controlados e não controlados

\begin{tabular}{lccc}
\hline Caracteristica & $\begin{array}{c}\text { Controlados } \\
(\mathbf{8})\end{array}$ & $\begin{array}{c}\text { Não } \\
\text { controlados (6) }\end{array}$ & p \\
\hline $\begin{array}{l}\text { Cortisol urinário } \\
\text { pré-operatório }\left(\mathrm{LSN}^{*}\right)\end{array}$ & $3,6 \pm 2,7$ & $4,8 \pm 4,1$ & 0,34 \\
ACTH pré-operatório $(\mathrm{pg} / \mathrm{dL})$ & $73,0 \pm 29,6$ & $115,5 \pm 128,1$ & 0,75 \\
Idade & $34,8 \pm 6,4$ & $24,0 \pm 9,4$ & 0,07 \\
Micro: macroadenoma & $5: 3$ & $4: 2$ & 1,0 \\
Microscopia: endoscopia & $4: 4$ & $2: 4$ & 0,13 \\
\hline
\end{tabular}

* LSN: cortisol livre urinário calculado em relação ao limite superior da (VN: $\leq 1,0)$.

Evolução dos resultados cirúrgicos na doença de Cushing: o índice de controle do hipercortisolismo pela cirurgia ( $\mathrm{n}$ controlados $/ \mathrm{n}$ operados) variou de $0(0 / 3)$ no ano 2001 a $1,0(2 / 2)$ no ano 2005 , correlacionando-se significativamente com o ano da cirurgia ao longo desses 5 anos ( $\mathrm{p}=0,01$; qui-quadrado) (Figura 4).

\section{Prolactinoma}

Casuística: foram avaliados 5 pacientes com prolactinoma, com idade média de $36 \pm 16,8$ anos (mediana: 32 anos; intervalo: 21-59 anos), sendo 3 do sexo feminino e 2 do sexo masculino. $\mathrm{Na}$ RM, todos apresentavam macroadenomas invasivos. Três já haviam sido submetidos à cirurgia por outras equipes (uma craniotomia fronto-orbitária e o restante via transesfenoidal). A indicação cirúrgica foi resistência à bromocriptina em 4 pacientes e intolerância à bromocriptina com crescimento tumoral em um paciente. Resistência foi caracterizada pelo uso de $15 \mathrm{mg} /$ dia ou mais de bromocriptina por pelo menos 3 meses sem normalização da prolactina ou redução do volume tumoral. O estudo anatomopatológico confirmou adenoma com imuno-histoquímica positiva para prolactina em todos.

Prolactina: no pré-operatório, na vigência de dose máxima de bromocriptina (15 a $25 \mathrm{mg} / \mathrm{dia}$ ), a prolactina foi $1895 \pm 3122 \mathrm{ng} / \mathrm{ml}$ (mediana: $410 \mathrm{ng} / \mathrm{ml}$; intervalo: 71-7424 ng/ml). Após a cirurgia, os níveis de prolactina foram reduzidos $(\mathrm{p}<0,001)$ para $583,4 \pm$ $928 \mathrm{ng} / \mathrm{ml}$ (mediana: $119 \mathrm{ng} / \mathrm{ml}$; intervalo: 14-2195 $\mathrm{ng} / \mathrm{ml}$ ), ainda em uso de bromocriptina (10-15 mg/ dia) (Figura 5).

Função adeno-hipofisária: entre os pacientes com cirurgia hipofisária prévia, dois já apresentavam pan-hipopituitarismo e mantiveram as mesmas deficiências hormonais. Nenhum paciente evoluiu com nova deficiência hormonal após a nova cirurgia.

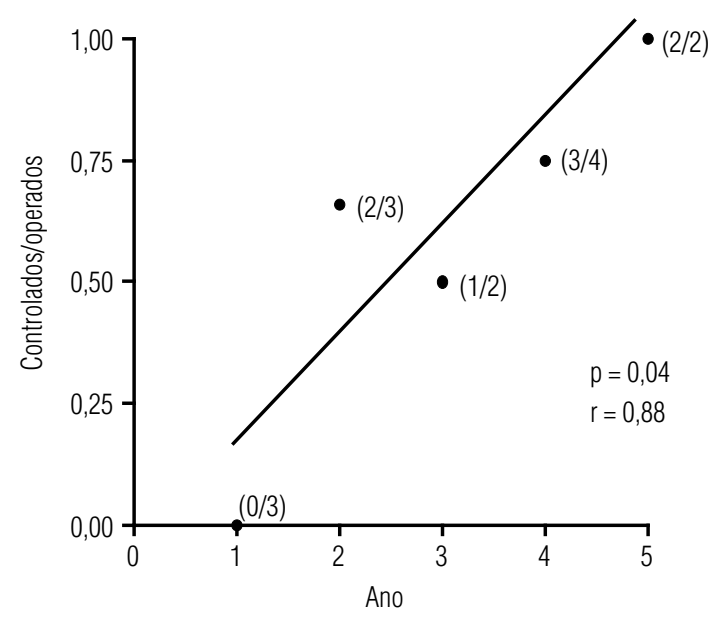

Figura 4. Evolução do índice de controle (controlados/operados) após a cirurgia transesfenoidal em 14 pacientes com doença de Cushing. 


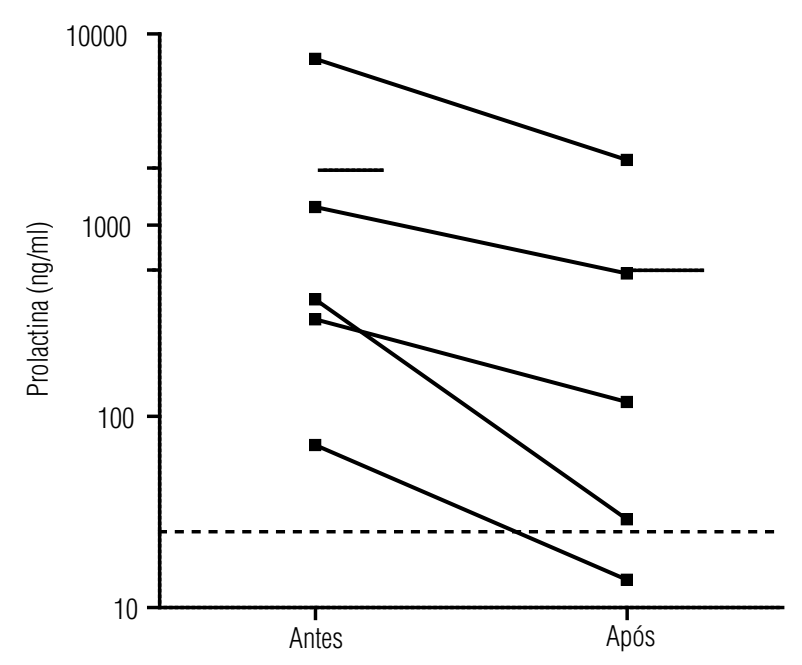

Figura 5. Níveis de prolactina $(\mathrm{ng} / \mathrm{ml})$ em pacientes portadores de macroprolactinoma antes e após cirurgia transesfenoidal (em uso de agonista dopaminérgico).

Visão: todos os pacientes apresentavam deficiência visual parcial antes da cirurgia. O procedimento cirúrgico não corrigiu ou piorou a deficiência em nenhum caso.

Complicações: nenhum paciente apresentou meningite, sangramento ou diabetes insípido. Um paciente apresentou fístula liquórica, corrigida com repouso e tamponamento nasal. Nenhum óbito ocorreu.

Controle hormonal: em 2 casos com resistência à bromocriptina $(40 \%)$, a prolactina se normalizou no pós-operatório e se manteve normal mesmo com redução das doses de bromocriptina (de 15-20 mg/dia para $10 \mathrm{mg} /$ dia em ambos).

\section{Adenoma secretor de TSH}

Caso: uma paciente de 19 anos, com tirotoxicose e bócio difuso, apresentou TSH de $34 \mathrm{mU} / \mathrm{L}$ (VN: 0,35$5,5 \mathrm{U} / \mathrm{dL}$ ); T4 livre de 3,3 ng/dL (VN: 0,7-1,54 ng/ $\mathrm{dL}$ ); T3 total de $1.132 \mathrm{ng} / \mathrm{dL}$ (VN: 75-220 ng/dL) e relação molar subunidade $\alpha / \mathrm{TSH}$ de 1,5 (VN: 1 ). A ressonância magnética mostrou um microadenoma de $0,8 \mathrm{~cm}$, do lado esquerdo, junto ao seio cavernoso. Não apresentava deficiência hormonal ou visual. Antes da cirurgia foi tratada com Sandostatin LAR, sem resposta clínica ou laboratorial satisfatória. Depois da cirurgia, apresentou melhora clínica importante, com redução do volume do bócio e dos níveis de TSH para $0,1 \mathrm{mU} / \mathrm{L}$, de T4 livre para $0,9 \mathrm{ng} / \mathrm{dL}$ e de T3 total para $130 \mathrm{ng} / \mathrm{dL}$, sendo considerada controlada. O exame anatomopatológico confirmou adenoma com imuno-histoquímica positiva para TSH. Evoluiu com hipo- tiroidismo central transitório, tendo sido tratada com levotiroxina por 4 meses. No sexto mês após a cirurgia, encontrava-se clinicamente eutiroidiana com TSH de $0,5 \mathrm{mUI} / \mathrm{L}$ e T4 livre de $1,2 \mathrm{ng} / \mathrm{dL}$.

Complicações: nenhuma complicação cirúrgica foi observada neste caso.

\section{DISCUSSÃO}

O presente trabalho analisou os resultados obtidos no tratamento cirúrgico dos adenomas hipofisários secretores pelo grupo de cirurgia hipofisária da Unifesp nos primeiros cinco anos de experiência (2001-2005). Experiência cirúrgica, de acordo com as diretrizes para o tratamento da acromegalia (5), é definida como: publicação dos resultados cirúrgicos em revistas indexadas contendo avaliação cirúrgica, bioquímica e seguimento adequado dos pacientes; treinamento em pelo menos 100 casos de cirurgia hipofisária e realização de pelo menos 25 cirurgias por ano.

Este grupo de cirurgiões realizou 180 cirurgias hipofisárias em 5 anos, resultando em média 36 cirurgias por ano. A maior parte das casuísticas cirúrgicas publicadas (PubMed) relata resultados de experiência acumulada em períodos mais longos, raramente se restringindo aos anos iniciais, em que os resultados são relativamente piores, o que dificulta uma comparação mais equilibrada entre nossos resultados e os dessas séries. Outros fatores de dificuldade nessa análise comparativa são a heterogeneidade dos critérios de "cura" ou de controle hormonal ao longo do tempo e a falta de consenso para esses critérios mesmo entre trabalhos contemporâneos.

Nessa avaliação, evitou-se a classificação dos resultados cirúrgicos em curados e não curados, posto que uma definição de cura oncológica seria necessariamente probabilística e dependente de um tempo mínimo de seguimento sem a doença. Além disso, essa dicotomia encarcera os resultados em apenas duas categorias e impede a avaliação de resultados cirúrgicos intermediários que certamente contribuem para o tratamento do paciente.

\section{Acromegalia}

Para a acromegalia, a definição de controle hormonal é controversa e os critérios têm variado com o passar do tempo. Níveis basais de GH, nadir de GH após glicose e IGF-I têm sido usados, isolados ou em conjunto, como critérios de controle por diferentes autores. Os primeiros relatos consideravam controlados os pacientes com $\mathrm{GH}<10 \mathrm{ng} / \mathrm{mL}(6)$, sendo que este valor vem de- 
crescendo progressivamente, acompanhando a evolução dos ensaios de $\mathrm{GH}$, mais específicos e com valores mais baixos do que os ensaios mais antigos. Por outro lado, vários estudos epidemiológicos têm demonstrado que a taxa de mortalidade associada à acromegalia tratada e com $\mathrm{GH} \leq 2,5 \mathrm{ng} / \mathrm{mL}$ se iguala à da população controle, e esse valor, também denominado GH seguro, tem sido frequentemente adotado como critério de controle (7-9). Também a normalização dos níveis de IGF-I em acromegálicos tem sido associada à redução da mortalidade em alguns estudos epidemiológicos (10). Além disso, valores de GH e IGF-I séricos que são discrepantes entre si, sobretudo com GH $<2-2,5 \mathrm{ng} / \mathrm{mL}$ e IGF-I elevada, são relatados em até $32 \%$ dos casos (11).

Atualmente, conforme sugerido pelo último consenso publicado em 2010 acerca dos critérios de cura na acromegalia (12), esses seriam normalização de IGF-I, GH basal $\leq 1,0 \mathrm{ng} / \mathrm{mL}$ e GH após sobrecarga oral de glicose $<0,3 \mathrm{ng} / \mathrm{mL}$. No presente estudo, foi usado como critério de controle o $\mathrm{GH}$ basal $\leq 2,5 \mathrm{ng} / \mathrm{mL}$ ( $\mathrm{GH}$ seguro) em função da sua validação epidemiológica, associado ou não à IGF-I normal para idade e sexo, visto que o objetivo do estudo foi avaliar a evolução dos resultados cirúrgicos ao longo do tempo.

Dessa forma, $36 \%$ de todos os tumores secretores de $\mathrm{GH}$ operados nesses cinco anos iniciais atingiram níveis seguros de $\mathrm{GH}(\leq 2,5 \mathrm{ng} / \mathrm{mL})$, sendo, portanto, considerados controlados pela cirurgia. Esse resultado inicial encontra-se abaixo dos $62 \%(\mathrm{n}=982$; intervalo: 42\%-76\%) encontrados numa metanálise de pacientes tratados por cirurgia transesfenoidal compilados de 11 casuísticas. A porcentagem de controle nos macroadenomas circunscritos observada em nossa casuística foi de $55 \%$, enquanto a de macroadenomas invasivos foi de apenas $20 \%$ e a de microadenomas, $40 \%$. Nessa mesma metanálise, $57 \%$ dos macroadenomas $(n=634$; 23\%-75\%), sem distinção entre circunscritos e invasivos, e $80 \%$ dos microadenomas $(\mathrm{n}=247$; intervalo: $71 \%$ $100 \%$ ) foram controlados pela cirurgia (13).

A importância do tempo de experiência nos resultados da cirurgia hipofisária transesfenoidal na acromegalia tem sido observada em diversos estudos (14-17). A evolução das taxas de sucesso cirúrgico em 139 pacientes operados por um único cirurgião em 21 anos mostrou que a taxa de remissão subiu de $48 \%$ nos primeiros oito anos (1974-1981) para 73\% no último período analisado (1991-1995). A análise comparativa entre o primeiro período e os períodos subsequentes (1981-1985, 19861990 e 1991-1995) mostrou melhora significativa dos resultados, enquanto a comparação entre esses períodos posteriores não mostra diferenças, sugerindo uma curva de aprendizado ascendente nos primeiros 5-10 anos de experiência que se estabiliza nos anos subsequentes (17). Assim, espera-se que nossos resultados cirúrgicos na acromegalia, passado esse período inicial, sejam significativamente melhores nos próximos anos.

\section{Doença de Cushing}

$\mathrm{Na}$ doença de Cushing, utilizamos a determinação do cortisol livre na urina de 24 horas, colhida após quatro ou mais semanas da cirurgia, como parâmetro de controle hormonal após a adenomectomia transesfenoidal. Esse procedimento foi considerado o mais adequado para avaliar o controle hormonal de nossos pacientes pelas seguintes razões: (I) reflete a produção diária de cortisol, (II) estava sempre elevado no diagnóstico desses pacientes, (III) foi também utilizado para avaliar a resposta ao tratamento pré-operatório com cetoconazol, (IV) foi realizado fora do período pós-operatório precoce, quando o efeito bloqueador do cetoconazol na síntese de cortisol ainda poderia estar presente (18) e (V) foi o único exame laboratorial disponível no pré e pós-operatório em todos os pacientes. Em contraste, outros métodos de avaliação, como o cortisol basal entre 1 e 7 dias após a cirurgia, bem como o aparecimento de sinais e sintomas de insuficiência adrenal nesse período, poderiam ser inadequados em pacientes pré-tratados com cetoconazol. A possibilidade de efeito residual da droga no pós-operatório favoreceria o encontro de níveis séricos de cortisol baixos em alguns pacientes, enquanto o desbloqueio do eixo corticotrófico durante o tratamento pré-operatório impediria o aparecimento de insuficiência adrenal em outros. Na presença de ambos os efeitos, alguns pacientes não controlados em avaliações mais tardias poderiam desenvolver insuficiência adrenal nesse período.

No presente estudo, $50 \%$ dos tumores secretores de ACTH operados nesses cinco anos iniciais foram considerados controlados, sendo $40 \%$ dos macroadenomas e $55 \%$ dos microadenomas. Entre as grandes séries cirúrgicas publicadas, os índices de remissão variam amplamente, entre $42 \%$ e $91 \%$ (19). A análise dos resultados de acordo com o ano de cirurgia mostrou aumento significativo da taxa de controle com o passar do tempo $(\mathrm{r}=$ $0,88 ; \mathrm{p}=0,04)$, demonstrando a existência de uma curva ascendente de aprendizado cirúrgico. Assim, é ilustrativo que, no primeiro ano, nenhum dos três pacientes tenha sido controlado pela cirurgia, ao passo que no último ano os dois pacientes operados tenham sido controlados. 
Diversos estudos têm demonstrado a importância do tempo de experiência nos resultados da cirurgia hipofisária transesfenoidal na doença de Cushing. A taxa de remissão após cirurgia transesfenoidal em 24 pacientes com doença de Cushing (18 microadenomas, 3 macroadenomas circunscritos e 3 macroadenomas invasivos) operados por Jules Hardy nos primeiros quinze anos de sua experiência (1961-1976), relatada em sua primeira publicação desses resultados (20), mostra uma taxa de remissão de $67 \%$ (16/24), sendo $72 \%$ (13/18) nos microadenomas, $100 \%(3 / 3)$ nos macroadenomas circunscritos e $0 \%(0 / 3)$ nos macroadenomas invasivos. Numa publicação posterior (21), com 75 pacientes (67 microadenomas, 4 macroadenomas circunscritos e 4 macroadenomas invasivos) operados entre 1963 e 1982, a taxa de remissão foi $84 \%(63 / 75)$, sendo $88 \%$ (58/67) nos microadenomas, $100 \%$ (4/4) nos macroadenomas circunscritos e $25 \%$ (1/4) nos macroadenomas invasivos.

Em 1984, Charles Wilson publicou uma revisão sobre a primeira década de sua experiência em microcirurgia hipofisária (22). Entre 1970 e 1982 foram operados, por via transesfenoidal, 1.000 adenomas hipofisários, sendo 774 adenomas funcionantes. Entre estes, 96 eram adenomas secretores de ACTH e 77\% (74/96) foram considerados controlados pela cirurgia. Entre os microadenomas, a porcentagem de controle foi $86 \%(63 / 73)$, enquanto nos macroadenomas foi $48 \%(11 / 23)$. Em trabalhos publicados recentemente pelo mesmo grupo, as porcentagens de controle da doença de Cushing são maiores. Em 2004 foi publicada uma série (23) com os resultados cirúrgicos de 200 casos de doença de Cushing, operados entre 1975 e 1998, sendo excluídos, portanto, os cinco primeiros anos de experiência. A porcentagem de controle hormonal foi $85 \%$ (169/200). Entre os microadenomas foi $86 \%$ (121/140), a mesma dos primeiros 10 anos de experiência. Já nos macroadenomas, a porcentagem aumentou para $80 \%(48 / 60)$, sendo $83 \%(43 / 52)$ nos macroadenomas circunscritos e $63 \%(5 / 8)$ nos macroadenomas invasivos.

Além da experiência cirúrgica, a identificação do adenoma no pré-operatório e a extensão do tumor são preditores importantes do sucesso cirúrgico na doença de Cushing $(24,25)$. Em nossa casuística, apenas um adenoma não foi identificado no pré-operatório e a porcentagem de macroadenomas (43\%) foi maior que a encontrada na literatura. Esses fatores, além da variabilidade na escolha dos critérios para definir controle da doença, podem explicar a variação das porcentagens de controle entre os diversos estudos.

A técnica cirúrgica empregada (microscopia ou endoscopia) não foi determinante para o aumento dos índices de controle hormonal na nossa casuística. $\mathrm{Na}$ maior série publicada de pacientes com doença de Cushing operados exclusivamente com auxílio endoscópico, os índices de remissão foram semelhantes aos da literatura (26).

\section{Prolactinoma}

Os adenomas hipofisários secretores de prolactina (prolactinomas) são os tumores hipofisários mais frequentes. A excelente resposta ao tratamento com agonistas dopaminérgicos, com normalização da prolactina em cerca de $90 \%$ dos casos e redução do tumor em cerca de $60 \%$ (27), faz dessa modalidade a primeira opção terapêutica para esses tumores. A intolerância ou resistência aos agonistas dopaminérgicos constituem as poucas indicações cirúrgicas para os prolactinomas. Os resultados cirúrgicos nos macroprolactinomas costumam ser insatisfatórios. Em macroprolactinomas invasivos, a cirurgia normaliza a prolactina em apenas $7 \%-50 \%$ dos casos $(28,29)$ e com elevada taxa de recorrência.

Nesse estudo, obtivemos melhora do controle hormonal em $40 \%$ dos casos, com normalização da prolactina com doses menores de bromocriptina daquelas usadas antes da cirurgia. Esse critério foi adotado porque todos os pacientes deste estudo retrospectivo permaneceram em uso de agonista dopaminérgico no pós-operatório. Na acromegalia, alguns estudos sugerem que a remoção incompleta do tumor melhora a resposta aos análogos da somatostatina $(30,31)$ e dados preliminares sugerem que o mesmo acontece com macroprolactinomas em relação aos agonistas dopaminérgicos (32). Nossos resultados corroboram essa hipótese.

\section{Adenoma secretor de TSH}

O adenoma hipofisário secretor de TSH é uma patologia rara, correspondendo a $0,5 \%$ a $2 \%$ dos tumores hipofisários. Nos últimos 20 anos, apenas cinco pacientes portadores de adenoma hipofisário secretor de TSH (tirotropinoma) foram acompanhados no Ambulatório de Neuroendocrinologia de Unifesp (33), sendo todos macroadenomas. Entre esses, 4 foram diagnosticados e operados antes de 2001 e nenhum deles foi controlado pela cirurgia. O único paciente portador de um adenoma secretor de TSH operado durante o período des- 
te estudo apresentava um microadenoma que obteve controle hormonal pela cirurgia. Numa extensa revisão da literatura publicada recentemente, dos 129 pacientes compilados, apenas $33 \%$ apresentaram controle hormonal após a cirurgia (34).

\section{Técnica cirúrgica}

O acesso endoscópico transnasal bilateral foi utilizado em 77\% dos casos deste estudo. Nesta técnica, o endoscópio é colocado por uma fossa nasal e manipulado pelo otorrinolaringologista durante todo o procedimento, enquanto os instrumentos cirúrgicos, colocados pela outra fossa nasal, são manipulados pelo neurocirurgião. O uso do endoscópio, além de facilitar o acesso por via transnasal, permite boa visibilidade da região selar durante todo o procedimento. A exploração da região com endoscópio, após a remoção do tumor, é também de grande importância, seja para identificar e remover restos tumorais, seja para procurar e corrigir possíveis lesões do diafragma selar que podem causar fístulas liquóricas. Esse procedimento exploratório aumenta a possibilidade de encontrar e ressecar resíduos tumorais não visualizados com microscópio cirúrgico. No entanto, está associado à maior incidência de fístulas liquóricas no intra-operatório $(35,36)$. Por ser uma técnica nova, não existem ainda evidências na literatura de que a melhor visualização da região selar pelo endoscópio resulte em maior chance de cura cirúrgica, em comparação com a utilização do microscópio $(26,37)$. No presente estudo não houve diferença estatística entre os resultados com essa técnica em relação à cirurgia com auxílio do microscópio.

\section{Complicações}

A taxa de mortalidade estimada na cirurgia hipofisária por via transesfenoidal é cerca de $1 \%$, de acordo com uma revisão de 30 séries cirúrgicas (38). Nenhum óbito peri-operatório ocorreu em nossa casuística. A fístula liquórica pós-operatória é uma complicação indesejada, por prolongar o tempo de internação e aumentar o risco de meningite. Nesta casuística, $10 \%$ dos casos evoluíram com fístula liquórica não complicada, sendo todas em macroadenomas. Nenhum caso teve indicação de nova abordagem cirúrgica ou meningite. A maioria dos autores relata índices menores dessa complicação, abaixo de 5\%. Esse fato pode ser explicado pela fase de aquisição de experiência da equipe cirúrgica. O sangramento nasal, presente em $7 \%$ dos casos, também foi maior que o relatado na literatura, em torno de $2 \%$. Todos os casos ocorreram em macroadenomas secretores de $\mathrm{GH}$ e foram resolvidos com medidas simples, como repouso e tamponamento nasal anterior, não sendo necessária reintervenção cirúrgica.

O desenvolvimento de nova deficiência hipofisária no pós-operatório é um critério importante na definição de sucesso cirúrgico. Neste estudo, todos os casos de diabetes insípido, $20 \%$ dos pacientes operados, foram transitórios. A incidência de diabetes insípido permanente após cirurgia transesfenoidal em outras séries é $1 \%$ a $2 \%$ $(37,38)$. Apesar da grande incidência de macroadenomas invasivos, o desenvolvimento de nova deficiência hormonal adeno-hipofisária aconteceu em apenas $9 \%$ dos casos, semelhante a outras séries $(13,39,40)$. Outra preocupação é a possibilidade de piora da perda visual por lesão do nervo óptico durante o procedimento cirúrgico. Nesta casuística, apenas um paciente $(2,5 \%)$ apresentou piora discreta do campo visual após a cirurgia.

\section{Melhora visual e hormonal}

O sucesso cirúrgico deve considerar, além do controle da hiperfunção hormonal, a possibilidade de melhora de uma deficiência hormonal ou perda visual preexistente. Nesta série, $45 \%$ dos pacientes com uma ou mais deficiências adeno-hipofisárias no pré-operatório ( $\mathrm{n}=$ 11) recuperaram pelo menos uma dessas funções após a cirurgia. Foram operados cinco pacientes com perda visual. Destes, 3 recuperaram totalmente $(60 \%)$ e 2 , parcialmente a visão (40\%).

Em conclusão, nossos resultados iniciais da cirurgia transesfenoidal de adenomas hipofisários secretores no controle hormonal refletem um período de aquisição de experiência. Os dados apresentados demonstram, na doença de Cushing, melhora progressiva dos níveis de cortisol urinário no pós-operatório inicial nos primeiros cinco anos, com taxas de complicações relativamente baixas. Assim, é provável que nos próximos anos os índices de controle serão melhores e comparáveis aos obtidos em centros especializados com maior tempo de experiência.

Declaração: os autores declaram não haver conflitos de interesse científico neste estudo.

\section{REFERÊNCIAS}

1. Schloffer H. Erfolgreiche operationen eines hypophysentumors auf nasalem wege. Wien Klin Wochenschr. 1907;20:621-4.

2. Cushing $H$. The Weir Mitchell lecture. Surgical experiences with pituitary adenoma. JAMA. 1914;63:1515-25. 
3. Hardy J. [Excision of pituitary adenomas by trans-sphenoidal approach]. Union Med Can. 1962;91:933-45.

4. Guiot G, Thibaut B, Bourreau M. [Extirpation of hypophyseal adenomas by trans-septal and trans-sphenoidal approaches]. Ann Otolaryngol Chir Cervicofac. 1959;76:1017-31.

5. Giustina A, Barkan A, Casanueva FF, Cavagnini F, Frohman L, Ho $\mathrm{K}$, et al. Criteria for cure of acromegaly: a consensus statement. $\mathrm{J}$ Clin Endocrinol Metab. 2000;85(2):526-9.

6. Tucker HS, Grubb SR, Wigand JP, Watlington CO, Blackard WG, Becker DP. The treatment of acromegaly by transsphenoidal surgery. Arch Intern Med. 1980;140(6):795-802.

7. Holdaway IM, Rajasoorya C. Epidemiology of acromegaly. Pituitary. 1999;2(1):29-41.

8. Ayuk J, Clayton RN, Holder G, Sheppard MC, Stewart PM, Bates AS. Growth hormone and pituitary radiotherapy, but not serum insulin-like growth factor-I concentrations, predict excess mortality in patients with acromegaly. J Clin Endocrinol Metab. 2004;89(4):1613-7.

9. Kaltsas GA, Isidori AM, Florakis D, Trainer PJ, Camacho-Hubner C, Afshar $F$, et al. Predictors of the outcome of surgical treatment in acromegaly and the value of the mean growth hormone day curve in assessing postoperative disease activity. J Clin Endocrinol Metab. 2001;86(4):1645-52.

10. Beauregard $C$, Truong $U$, Hardy J, Serri O. Long-term outcome and mortality after transsphenoidal adenomectomy for acromegaly. Clin Endocrinol (Oxf). 2003;58(1):86-91.

11. Costa AC, Rossi A, Martinelli CE Jr, Machado HR, Moreira AC. Assessment of disease activity in treated acromegalic patients using a sensitive GH assay: should we achieve strict normal GH levels for a biochemical cure? J Clin Endocrinol Metab. 2002;87(7):3142-7.

12. Giustina $A$, Chanson $P$, Bronstein $M D$, Klibanski $A$, Lamberts $S$, Casanueva FF, et al. A consensus on criteria for cure of acromegaly. J Clin Endocrinol Metab. 2010;95:3141-8.

13. Boeving A, Borba LA, Rodrigues AM, Orichowski EB, Paz Filho GJ, Santos $\mathrm{CM}$, et al. [Outcome of surgical treatment for acromegaly performed by a single neurosurgeon and cumulative meta-analysis]. Arq Bras Endocrinol Metabol. 2006;50(5):884-92.

14. Gittoes NJ, Sheppard MC, Johnson AP, Stewart PM. Outcome of surgery for acromegaly--the experience of a dedicated pituitary surgeon. QJM. 1999;92(12):741-5.

15. Lissett CA, Peacey SR, Laing I, Tetlow L, Davis JR, Shalet SM. The outcome of surgery for acromegaly: the need for a specialist pituitary surgeon for all types of growth hormone $(\mathrm{GH})$ secreting adenoma. Clin Endocrinol (Oxf). 1998;49(5):653-7.

16. Erturk E, Tuncel E, Kiyici S, Ersoy C, Duran C, Imamoglu S. Outcome of surgery for acromegaly performed by different surgeons: importance of surgical experience. Pituitary. 2005;8(2):93-7.

17. Ahmed S, Elsheikh M, Stratton IM, Page RC, Adams CB, Wass JA. Outcome of transphenoidal surgery for acromegaly and its relationship to surgical experience. Clin Endocrinol (Oxf). 1999;50(5):561-7.

18. Sonino N, Boscaro M, Paoletta A, Mantero F, Ziliotto D. Ketoconazole treatment in Cushing's syndrome: experience in 34 patients. Clin Endocrinol (Oxf). 1991;35(4):347-52.

19. Bochicchio D, Losa M, Buchfelder M. Factors influencing the immediate and late outcome of Cushing's disease treated by transsphenoidal surgery: a retrospective study by the European Cushing's Disease Survey Group. J Clin Endocrinol Metab. 1995;80(11):3114-20.

20. Bigos ST, Somma M, Rasio E, Eastman RC, Lanthier A, Johnston $\mathrm{HH}$, et al. Cushing's disease: management by transsphenoidal pituitary microsurgery. J Clin Endocrinol Metab. 1980;50(2):348-54.

21. Hardy J. Presidential address: XVII Canadian Congress of Neurological Sciences. Cushing's disease: 50 years later. Can J Neurol Sci. 1982;9(4):375-80.
22. Wilson CB. A decade of pituitary microsurgery. The Herbert Olivecrona lecture. J Neurosurg. 1984;61(5):814-33.

23. Hammer GD, Tyrrell JB, Lamborn KR, Applebury CB, Hannegan $E T$, Bell S, et al. Transsphenoidal microsurgery for Cushing's disease: initial outcome and long-term results. J Clin Endocrinol Metab. 2004;89(12):6348-57.

24. Hoybye $\mathrm{C}$, Grenback E, Thoren M, Hulting AL, Lundblad L, von Holst $\mathrm{H}$, et al. Transsphenoidal surgery in Cushing disease: 10 years of experience in 34 consecutive cases. J Neurosurg. 2004;100(4):634-8.

25. Newell-Price J, Grossman A. Diagnosis and management of Cushing's syndrome. Lancet. 1999;353(9170):2087-8.

26. Netea-Maier RT, van Lindert EJ, den Heijer M, van der Eerden A, Pieters GF, Sweep CG, et al. Transsphenoidal pituitary surgery via the endoscopic technique: results in 35 consecutive patients with Cushing's disease. Eur J Endocrinol. 2006;154:675-84.

27. Colao A, Di Sarno R, Sarnacchiaro F, Ferone D, Di Renzo G, Merola $\mathrm{B}$, et al. Prolactinomas resistant to standart dopamin agonists respond to chronic cabergoline treatment. J Clin Endocrinol Metab. 1997;82:876-83.

28. Molitch ME. Medical treatment of prolactinomas. Endocrinol Metab Clin North Am. 1999;28:143.

29. Gukalp HZ, Deda H, Attar A, U ur HC, Arasil E, Egemen N. The neurosurgical management of prolactinomas. J Neurosurg Sci. 2000;44(3):128-32.

30. Colao A, Attanasio R, Pivonello R, Cappabianca P, Cavallo LM, Lasio $G$, et al. Partial surgical removal of growth hormone-secreting pituitary tumors enhances the response to somatostatin analogs in acromegaly. J Clin Endocrinol Metab. 2006;91(1):85-92. Epub 2005 Nov

31. Karavitaki N, Turner HE, Adams CB, Cudlip S, Byrne JV, Fazal-Sanderson $\mathrm{V}$, et al. Surgical debulking of pituitary macroadenomas causing acromegaly improves control by lanreotide. Clin Endocrinol (Oxf). 2008;68(6):970-5. Epub 2007 Nov 19.

32. Iguchi $D$, Glezer A, Cescato VAS, et al. Does partial surgery removal of macroprolactinomas improve their response to dopamine agonists treatment?The Endocrine Society, 90th Annual Meeting, 2008. OR27-1;111.

33. Barbosa ER, Oliveira JHA, Abucham J. Adenomas hipofisários secretores de TSH: revisão de casuística. Arq Bras Endocrinol Metab. 2005;49:S105.

34. Beck-Peccoz P, Persani L. Medical management of thyrotropin-secreting pituitary adenomas. Pituitary. 2002;5(2):83-8.

35. Baussart B, Aghakhani N, Portier F, Chanson P, Tadie M, Parker F. [Endoscope-assisted microsurgery for invasive endo- and suprasellar pituitary macroadenomas: a consecutive retrospective study with 13 patients]. Neurochirurgie. 2005;51(5):455-63.

36. Santos R, Zymberg S, Abucham J, Gregório L, Weckx L. Acesso endoscópico transnasal aos tumores selares. Rev Bras Otorrinolaringol. 2007;73(4):463-75.

37. Yang I, Wang MB, Bergsneider M. Making the transition from neurosurgery to endoscopic trans-sphenoidal pituitary neurosurgery. Neurosurg Clin N Am. 2010;21(4):643-51.

38. Laws ER Jr, Thapar K. Pituitary surgery. Endocrinol Metab Clin North Am. 1999;28(1):119-31.

39. Nomikos P, Fahlbusch R, Buchfelder M. Recent developments in transsphenoidal surgery of pituitary tumors. Hormones (Athens). 2004;3(2):85-91.

40. Sheaves $R$, Jenkins $P$, Blackburn $P$, Huneidi AH, Afshar F, Medbak $S$, et al. Outcome of transsphenoidal surgery for acromegaly using strict criteria for surgical cure. Clin Endocrinol (Oxf). 1996;45(4):407-13. 